\title{
PERSPECTIVE
}

\section{Real-time Ultrasound Fusion Imaging-Guided Interventions: a Review}

\author{
CPY Chien, KH Lee, V Lau \\ Department of Radiology, Queen Mary Hospital, Hong Kong
}

\begin{abstract}
Ultrasound fusion imaging is a novel technique that allows the fused synchronous display of computed tomography or magnetic resonance images during real-time ultrasound scanning. It has been widely applied in various ultrasoundguided interventions to enhance lesion detectability, thereby improving procedural accuracy and safety. In this article, we describe the current status and our institutional experience of the application of ultrasound fusion imaging in hepatobiliary, renal, and musculoskeletal interventions. We also discuss techniques, challenges, and recommendations for ultrasound fusion-guided interventions.
\end{abstract}

Key Word: Biopsy

\section{中文摘要}

\section{實時超聲融合成像引導介入綜述}

\author{
錢珮恩、李錦浩、劉泳恒
}

\begin{abstract}
超聲融合成像是一種新技術，允許在實時超聲掃描期間同步融合顯示電腦斷層掃描或磁共振圖像。 這種新技街已被廣泛應用於各種超聲引導介入, 以提高病變檢出, 從而提高手術的準確性和安全 性。本文描述超聲融合成像在肝膽、腎臟和肌肉骨骼介入中應用的現狀和我們機構的經驗。我們討 論超聲融合引導介入的技術、挑戰和建議。
\end{abstract}

\footnotetext{
Correspondence: Dr CPY Chien, Department of Radiology, Queen Mary Hospital, Hong Kong

Email: chienpyc@gmail.com

Submitted: 2 Dec 2020; Accepted: 22 Feb 2021

Contributors: All authors designed the study. CPYC acquired the data. All authors analysed the data, drafted the manuscript, and critically revised the manuscript for important intellectual content. All authors had full access to the data, contributed to the study, approved the final version for publication, and take responsibility for its accuracy and integrity.
}

Conflicts of Interest: All authors have no conflicts of interest to declare.

Funding/Support: This research received no specific grant from any funding agency in the public, commercial, or not-for-profit sectors.

Ethics Approval: This study was approved by Hong Kong West Cluster Research Ethics Committee and the requirement to obtain informed consent was waived (IRB Ref UW19-267).

Disclosure: This topic was presented at the European Congress of Radiology 2020, 15-19 Jul 2020, Vienna, Austria (Poster C-03874).

Acknowledgement: We thank Dr Tina PW Lam for general supervision and administrative support throughout the project. 


\section{INTRODUCTION}

Ultrasound is a widely used imaging modality to guide percutaneous interventional procedures due to its easy accessibility, real-time capability, and lack of radiation. During ultrasound-guided interventions, interventional radiologists often localise the target lesion and carry out procedures by cognitive fusion with reference to the computed tomography $(\mathrm{CT})$ or magnetic resonance (MR) images. Despite advancements in ultrasound technology, visualisation of the target lesion can be difficult due to isoechogenicity with background parenchyma, obscuration by gas/calcification-related posterior acoustic shadowing, or attenuation of the ultrasound beam in obese patients. ${ }^{1}$

Medical image fusion is defined as the registration and overlaying of images from the same or different imaging modalities. Ultrasound fusion offers the opportunity of better localisation by displaying CT/MR images with real-time ultrasound side-by-side in the same plane and position. ${ }^{2,3}$ It improves the accuracy and confidence of the interventional radiologists during procedures. ${ }^{3}$

Real-time ultrasound fusion-guided intervention has gained popularity in recent years. Previous studies have evaluated the clinical applications of real-time ultrasound image fusion in different anatomical regions including liver, kidney, pancreas, breast, prostate and musculoskeletal system. ${ }^{2-8}$ Contrast-enhanced ultrasound (CEUS) can be used as an adjunct to further increase the sensitivity of lesion detection. ${ }^{9}$

In this review, we describe the real-time ultrasound fusion imaging technique used in our institution, and our experience of applications of ultrasound fusion imaging in hepatobiliary, renal, and musculoskeletal interventions. We also discuss the challenges associated with ultrasound fusion-guided interventions and provide recommendations.

\section{REAL-TIME ULTRASOUND FUSION IMAGING TECHNIQUE}

There are several tracking methods for ultrasound probes, including electromagnetic, optical, and image-based. ${ }^{2}$ In our institution, an electromagnetic tracking system is used as the tracking method for ultrasound fusionguided percutaneous interventions (Figure 1). The tracking system consists of a magnetic field transmitter, a position sensor, and a position sensor unit. The magnetic field transmitter, which is positioned next to the patient, creates a position-varying magnetic field. This induces electric currents in the position sensor mounted on the ultrasound transducer. During ultrasound probe movement, information regarding the magnitude of the induced current in the position sensor, which changes with the magnetic field strength, is transmitted back to the position sensor unit of the ultrasound machine, enabling tracking of probe position relative to the magnetic field transmitter.

To start the fusion procedure, the CT or MR images best depicting the target lesion and its anatomic relationship are uploaded to the ultrasound system. Next, coregistration-the process of overlaying realtime ultrasound and CT/MR images-is performed by using either external fiducial markers or internal anatomic landmarks. External fiducial markers contain position sensors, which allow automatic ultrasound image fusion when placed to the body surface close to the target organ when performing CT/MR. For internal

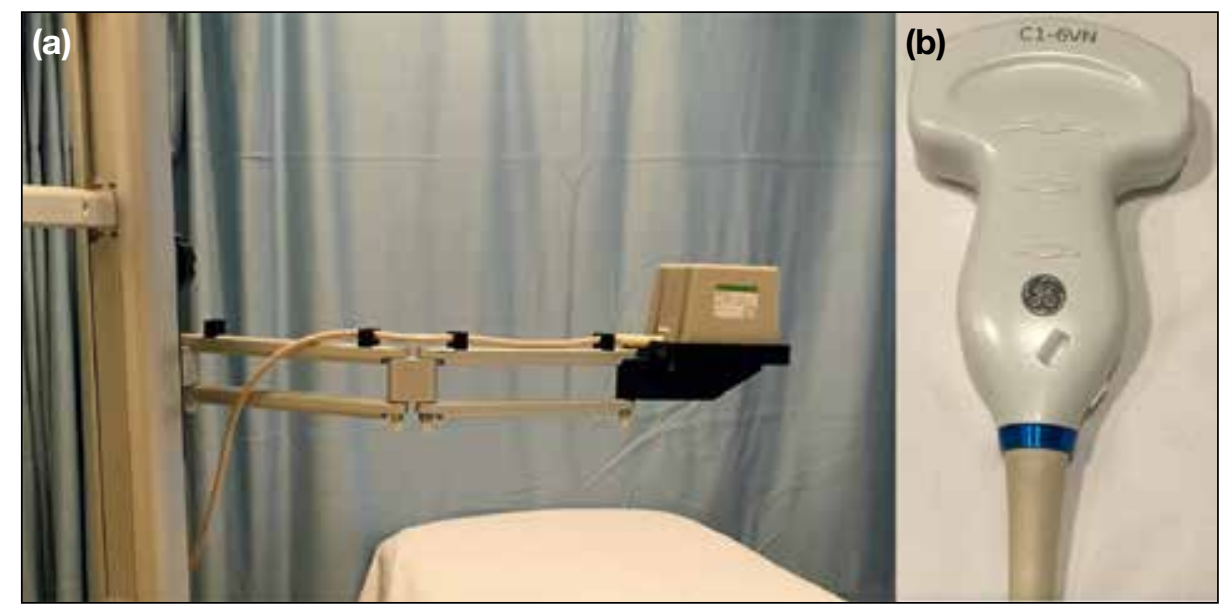

Figure 1. Electromagnetic tracking system for fusion imaging technique. (a) The magnetic field transmitter is mounted on support stand next to the patient. (b) The ultrasound transducer with the position sensor mounted on it, which is used for registration and throughout the intervention process. 
anatomic landmarks, plane and point registrations are performed manually (Figure 2). Plane registration is performed by aligning the ultrasound probe in the same plane (usually the axial plane) as the uploaded CT/MR images. Point registration is performed by marking standardised anatomical landmarks within the target organ/area (e.g., vessels, calcifications, cysts) manually on both the ultrasound and CT/MR images. Finally, the operator should check if accurate registration has been achieved by scrutinising the region of interest, including the target lesion and its surrounding anatomic structures. The process of point registration can be repeated until optimal registration is obtained. After coregistration, the $\mathrm{CT} / \mathrm{MR}$ images are displayed on the monitor side-by-side with the real-time ultrasound images in a synchronous manner and updated simultaneously according to the change in position and imaging plane of ultrasound probe.

To avoid registration errors, movement of the transmitter and patient should be avoided after coregistration. Therefore, a stable and comfortable body position should be ensured to minimise patient movement. A short time interval between the CT/MR examination and the interventional procedure is also preferred to minimise interval changes in anatomy. In our institution, we usually perform the ultrasound fusion-guided interventions within 1 to 2 months after acquisition of the CT/MR images.

\section{APPLICATIONS OF FUSION IMAGING \\ Hepatobiliary Interventions}

Common ultrasound-guided hepatobiliary interventions include lesion biopsy, tumour ablation, and abscess drainage. Deep, small and isoechoic liver lesions are difficult to be visualised in ultrasound. Synchronous visualisation of both real-time ultrasound and corresponding $\mathrm{CT} / \mathrm{MR}$ images improves diagnostic and therapeutic accuracy. Park et $\mathrm{al}^{10}$ reported that ultrasound fusion allowed accurate localisation of target lesion in the cirrhotic liver and decreased false sampling of pseudolesion in the background of coarsened parenchymal echogenicity.

Tumours located near the hepatic dome are technically challenging to ablate due to the deep location and suboptimal ultrasound visualisation, resulting in increased risk of thermal injury to lung and diaphragm and incomplete ablation. ${ }^{11,12}$ Lee et al $^{13}$ demonstrated that ultrasound fusion with CT/MR images could reduce false-positive detection for lesions $<2 \mathrm{~cm}$, and enhanced lesion detectability of small hepatocellular carcinoma for percutaneous radiofrequency ablation. Song et $\mathrm{al}^{14}$ showed that fusion imaging improved the conspicuity of hepatocellular carcinoma and feasibility of ablation of tumours that were not identifiable on ultrasound alone. The authors found that 26 out of 82 tumours poorly seen on fusion imaging could still be ablated by placing the electrode based on peritumoral anatomical landmarks. ${ }^{14}$

In hepatobiliary fusion imaging, the images/sequences best showing the target lesion is used for coregistration, such as arterial phase for hypervascular mass and portovenous phase for hypovascular mass on CT and hepatobiliary phase on MR imaging. The common anatomical landmarks used for registration include vascular bifurcations, such as right hepatic vein-inferior vena cava junction and portal vein bifurcation (Figure 3), or non-index lesions, such as liver cysts.

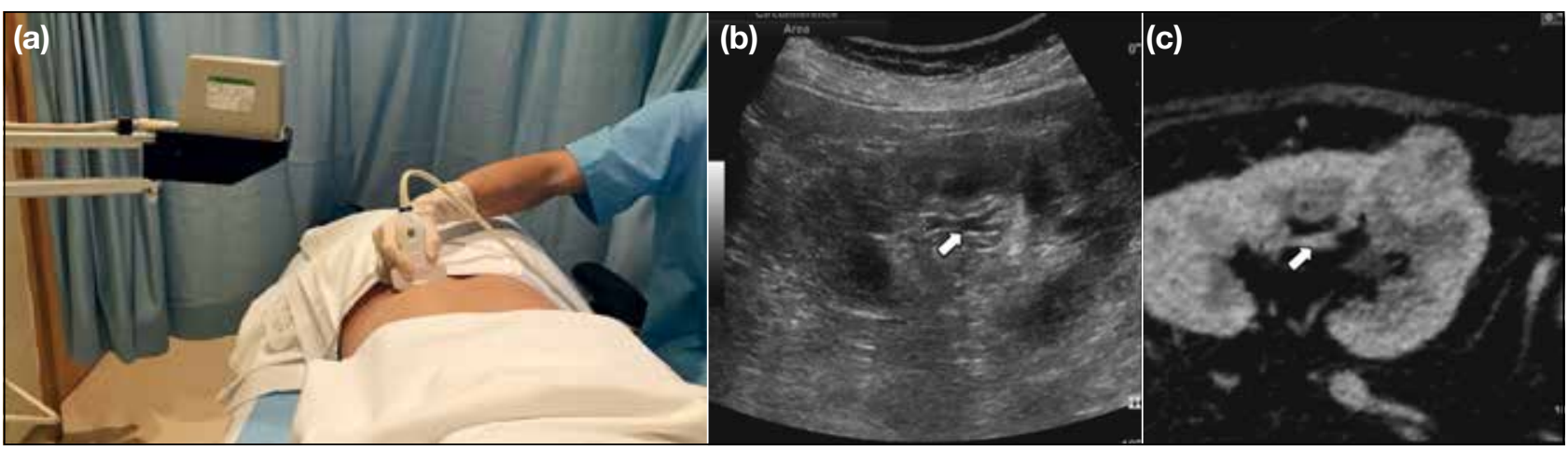

Figure 2. Steps of ultrasound-computed tomography (CT) coregistration. (a) Photograph showing plane registration performed by aligning the ultrasound probe in the same axial plane as the CT of the kidney with patient in prone position in kidney examination. (b) Ultrasound and (c) corresponding CT images showing point registration performed by marking the same anatomical point-bifurcation of the main renal artery into the superior and inferior segmental branches (arrows). 
In our experience, fusion imaging improves the detection of deep and small isoechoic lesions in the liver (Figure 4), hence reducing sampling errors during biopsy. In patients with hepatocellular carcinoma who have previously received transarterial chemoembolisation, ultrasound fusion allows more accurate localisation of active residual or recurrent disease in the background of echogenic parenchymal/tumoral lipiodol uptake
(Figure 5). This technique also allows ablation of small and difficult lesions to be done under real-time ultrasound instead of CT guidance, potentially reducing radiation exposure to patients and radiologists.

\section{Pancreatic Interventions}

The pancreas, especially the pancreatic tail, is often obscured by overlying bowel gas on ultrasound owing
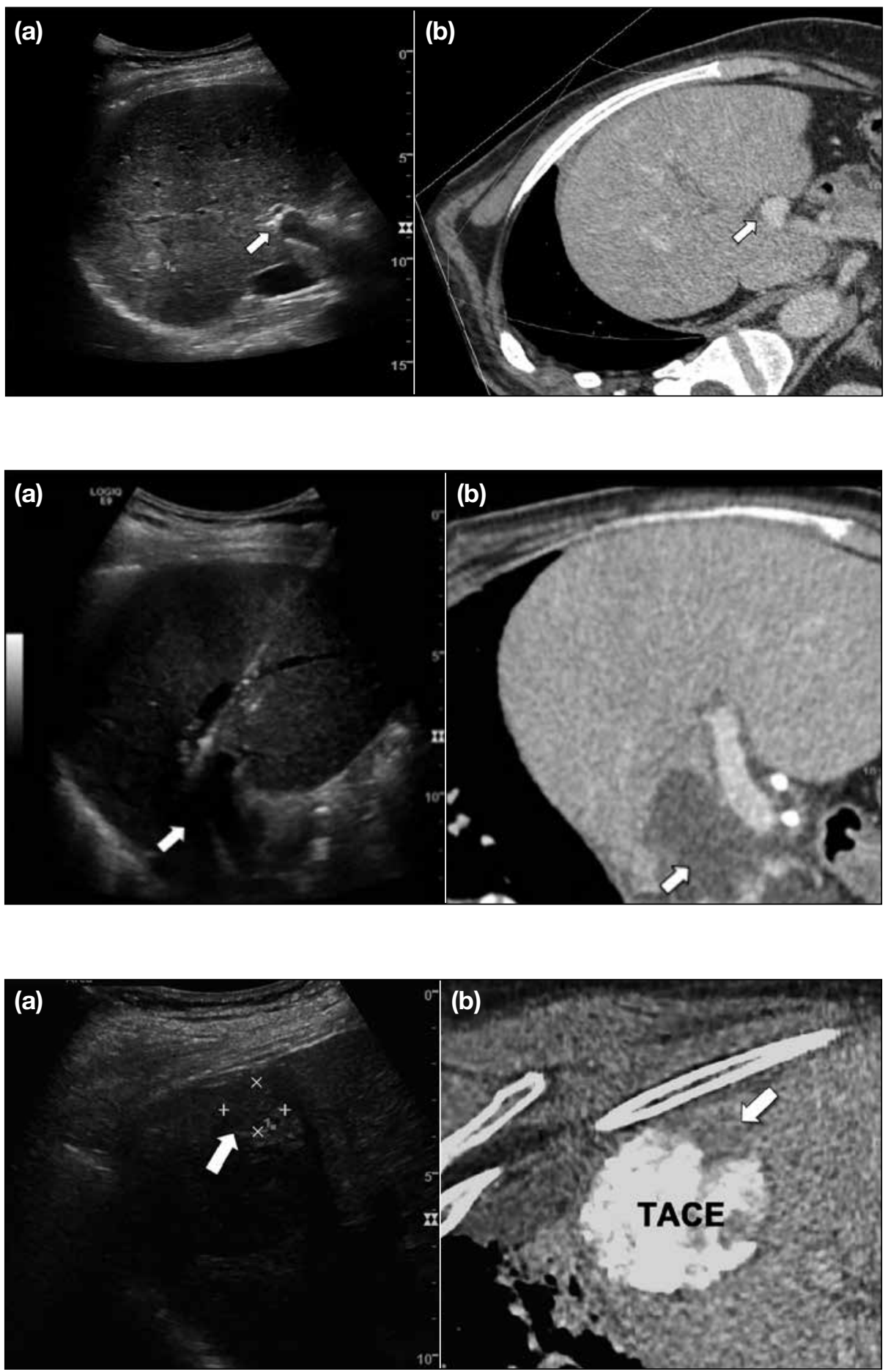

Figure 3. (a) Ultrasound and (b) corresponding computed tomography images showing point registration performed by marking the bifurcation of main portal vein into the left and right branch of portal vein (arrows) in liver examination.
Figure 4. Coregistered (a) ultrasound and (b) venous phase computed tomography images of liver hilum lesion for biopsy (arrows). The lesion was inconspicuous on ultrasound image alone.
Figure 5. Coregistered (a) ultrasound and (b) venous phase computed tomography images of a middle-aged female patient with hepatocellular carcinoma posttransarterial chemoembolisation for radiofrequency ablation of the residual tumour (white arrows). The residual tumour was isoechoic to the lipoidal stained region on ultrasound images. 
to its retroperitoneal location. CT or MR are better modalities for assessment of the pancreas and its relationship with adjacent structures such as the stomach, duodenum, portal vein, aorta, and celiac axis. Therefore, combining ultrasound and CT/MR allows real-time visualisation of the needle path and increases the accuracy during interventions. Zhang et $\mathrm{al}^{15}$ reported that applying ultrasound-CT fusion in percutaneous drainage of walled-off necrosis was associated with fewer complications and a higher success rate when compared with ultrasound alone, resulting in shorter hospital stay and lower costs. To the best of our knowledge, there is no evidence on ultrasound fusion-guided interventions of pancreatic tumours in the literature, likely because the deep retroperitoneal location of the pancreas renders percutaneous access difficult.

\section{Renal Interventions}

Ultrasound is good for discriminating solid renal lesions from cystic lesions. However, it has limitations in the detection and characterisation of solid lesions, especially when the lesion is isoechoic to the renal parenchyma. ${ }^{16} \mathrm{Up}$ to $35 \%$ of small $(<3 \mathrm{~cm})$ renal cell carcinomas are isoechoic to renal parenchyma on ultrasound.$^{17}$ Helck et al ${ }^{18}$ reported that ultrasound image fusion improved the identifiability and assessment of renal lesions compared with other modalities. Another study found improved image-guided tumour ablation in the ultrasound fusion-guided treatment of patients with renal tumours, particularly for lesions $<4 \mathrm{~cm}$ and in patients with a solitary kidney, in terms of avoiding surgical nephrectomy and preserving renal function. ${ }^{19}$ Andersson et $\mathrm{al}^{20}$ reported improved outcomes with ultrasound-CT fusion guidance compared with ultrasound alone in radiofrequency ablation of small renal masses.

In renal ultrasound fusion, the patient is usually positioned in the lateral decubitus or prone position. The images/ sequences best showing the target lesion are used for registration, such as arterial and nephrographic phases on CT imaging, or T1-weighted post-contrast sequences on MR imaging. Standardised anatomical landmarks used for registration include the bifurcation of the main renal artery into the superior and inferior segmental branches, or the confluence of the superior and inferior segmental renal veins into the main renal vein. Stable non-target lesions such as renal cysts or calcifications could also be used for registration.

In our experience, ultrasound fusion imaging guidance is particularly useful in the biopsy of isoechoic and upper pole renal lesions, which are difficult to localise on greyscale ultrasound (Figure 6a and b). Fusion imaging also helps to target the most appropriate part of the cystic lesion to be biopsied and to identify the best location for electrode placement during ablation. In patients with Von Hippel-Lindau syndrome, ultrasound fusion helps to identify renal cell carcinomas for radiofrequency ablation in the background of multiple renal cysts (Figure $6 \mathrm{c}$ and $\mathrm{d})$. Furthermore, fusion imaging helps determine the correct path for the needle, to avoid damaging the renal vessels and pelvis.

\section{Musculoskeletal Interventions}

In addition to abdominal applications, real-time ultrasound fusion imaging guidance is helpful in various musculoskeletal interventions, including therapeutic injections and biopsy of mass and non-mass lesions. ${ }^{5,6}$ Klauser et $\mathrm{al}^{8}$ demonstrated the feasibility of using ultrasound-CT fusion in therapeutic sacroiliac joints injections in patients with chronic sacroiliitis, obviating the need for repeated radiation exposure in young patients with spondyloarthropathies who needed repeated injections. Furthermore, Burke et $\mathrm{al}^{6}$ reported the use of ultrasound fusion in therapeutic injections of the pudendal nerve, piriformis muscle, and sacroiliac joints, as well as barbotage for calcific tendinopathy.

Fusion imaging complements ultrasound with augmented anatomical details demonstrated on axial imaging, thereby helping the operators plan the trajectory and avoid critical structures such as neurovascular bundles during soft tissue mass biopsy. Furthermore, lesions poorly visualised on ultrasound, such as non-fatty components of lipomatous tumours and areas of active muscle oedema in patients with suspected myopathy, can be targeted with confidence by side-by-side correlation between real-time ultrasound and MR images. Van De Vlekkert et $\mathrm{al}^{21}$ demonstrated that using MR to select target muscle with active oedema for biopsy decreased the false-negative sampling rate from $23 \%$ to $19 \%$ in patients with suspected idiopathic inflammatory myopathy. Lee et $\mathrm{al}^{5}$ demonstrated the feasibility and benefits of performing muscle biopsy under ultrasoundMR fusion imaging guidance in the patients with suspected myopathy, by sampling site of active muscle oedema without significant fatty infiltration. Our centre currently employs ultrasound-MR fusion when performing muscle biopsy for suspected myopathy (Figure 7), obviating the need for open surgical biopsy, which is more invasive and creates a larger wound. During the procedure, fat-suppressed T2-weighted 

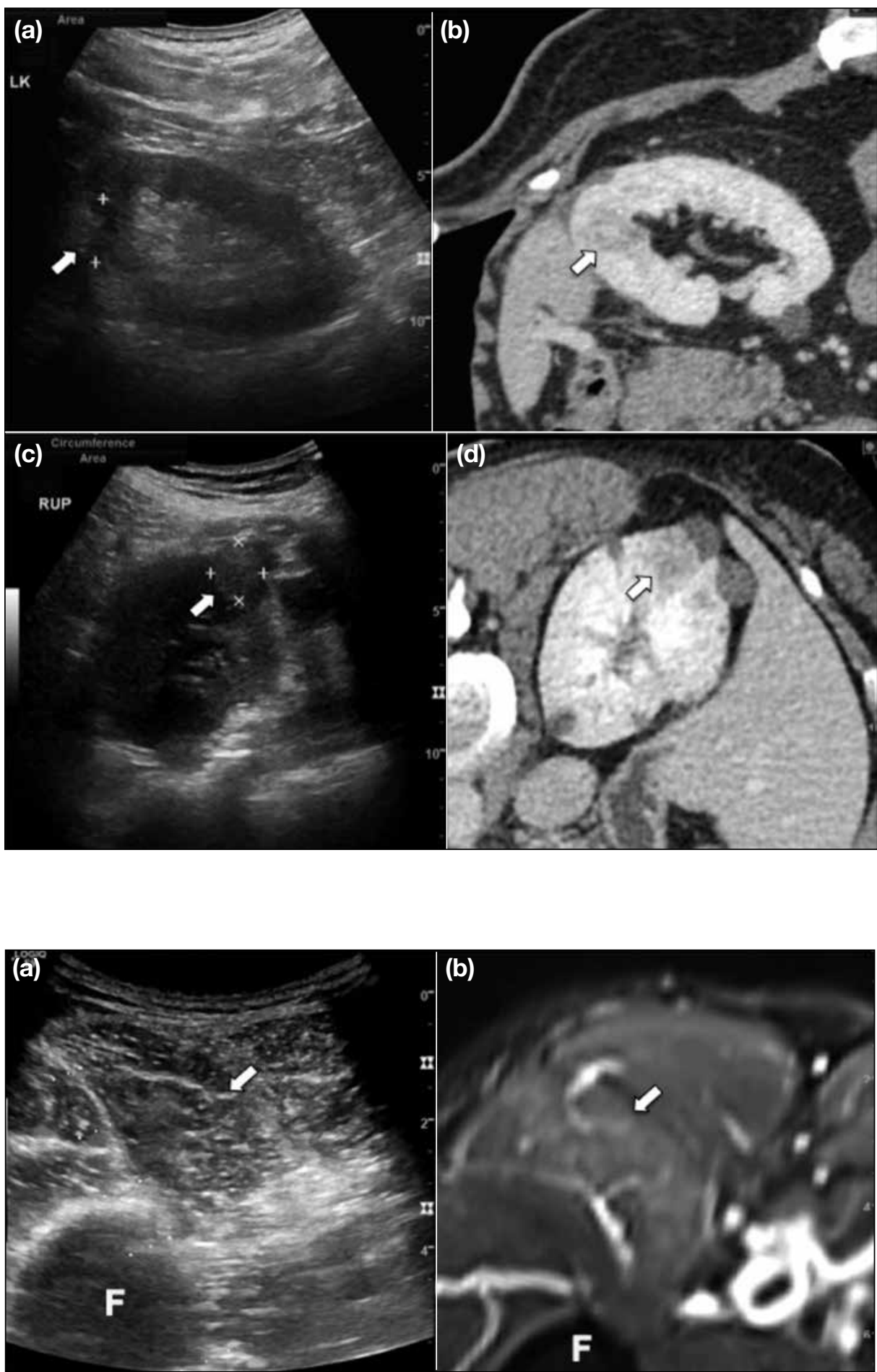

Figure 7. Coregistered (a) ultrasound and (b) T2-weighted magnetic resonance images of the left thigh muscles and left femur $(F)$ showing high T2-weighted signal intensity in the short head of bicep femoris muscle (arrows) but normal ultrasound appearance. images of the pelvis and thighs are used for image fusion to target the area of active muscle inflammation. Standardised anatomical landmarks for point registration include the saphenofemoral junction, common femoral artery bifurcation, and quadriceps tendon insertion at the superior pole of the patella.

\section{CONTRAST-ENHANCED ULTRASOUND}

There are additional benefits of CEUS in abdominal imaging, including improved detectability and conspicuity of liver and renal lesions. ${ }^{22,23}$ Because CEUS allows real-time visualisation of contrast enhancement 
of the septum and nodules in cystic renal tumours, the most representative part of the lesion can be identified for biopsy or ablation. ${ }^{22}$ Meloni et $\mathrm{al}^{23}$ demonstrated the use of CEUS in percutaneous treatment of hepatic and renal tumours, including immediate visualisation and real-time assessment of the ablation results.

In our experience, CEUS is particularly useful when the target lesion is small, isoechoic to the parenchyma, and posteriorly located in the liver (Figure 8). Ultrasound contrast can be injected during ablation screening for tumour detection, as well as during the ablation process (Figure 9). This further increases the interventional radiologist's confidence and accuracy when performing ablation in inconspicuous lesions.

\section{CHALLENGES AND RECOMMENDATIONS}

There are some possible technical limitations to real-time ultrasound fusion imaging techniques. First, ultrasound fusion may potentially prolong the procedure time owing to the extra time needed for image coregistration. However, in our experience, the image fusion step is simple and usually takes $<10$ minutes. Moreover, ultrasound fusion guidance decreases the time needed for trajectory planning, alleviating the need for re-positioning of the needle or ablation electrode. Thus, operator confidence and accuracy are increased and procedure time is shortened. Second, misregistration of images can occur owing to patient movement, uncooperative breathing movements, and tissue deformation by probe compression. ${ }^{2}$ One study showed that the mean maximum registration error between real-time ultrasound and fused CT images was $11.5 \mathrm{~mm} .{ }^{24}$ To minimise image misregistration, plane registration should be performed in the same imaging plane as the CT/MR examinations; patient movement should be avoided after the image coregistration; and the operator should maintain a steady and gentle force on the ultrasound probe throughout the procedure to reduce the tissue deformation, which is particular challenging in superficial lesions. Third, the time interval between the CT/MR examination and real-time ultrasound fusion-guided intervention can affect the accuracy of coregistration owing to disease progression or change in patient's body habitus. Therefore, it is crucial to use upto-date CT/MR images for coregistration.

\section{CONCLUSION}

In conclusion, real-time ultrasound fusion imaging is a useful tool in various interventional procedures, including the abdominal and musculoskeletal systems.
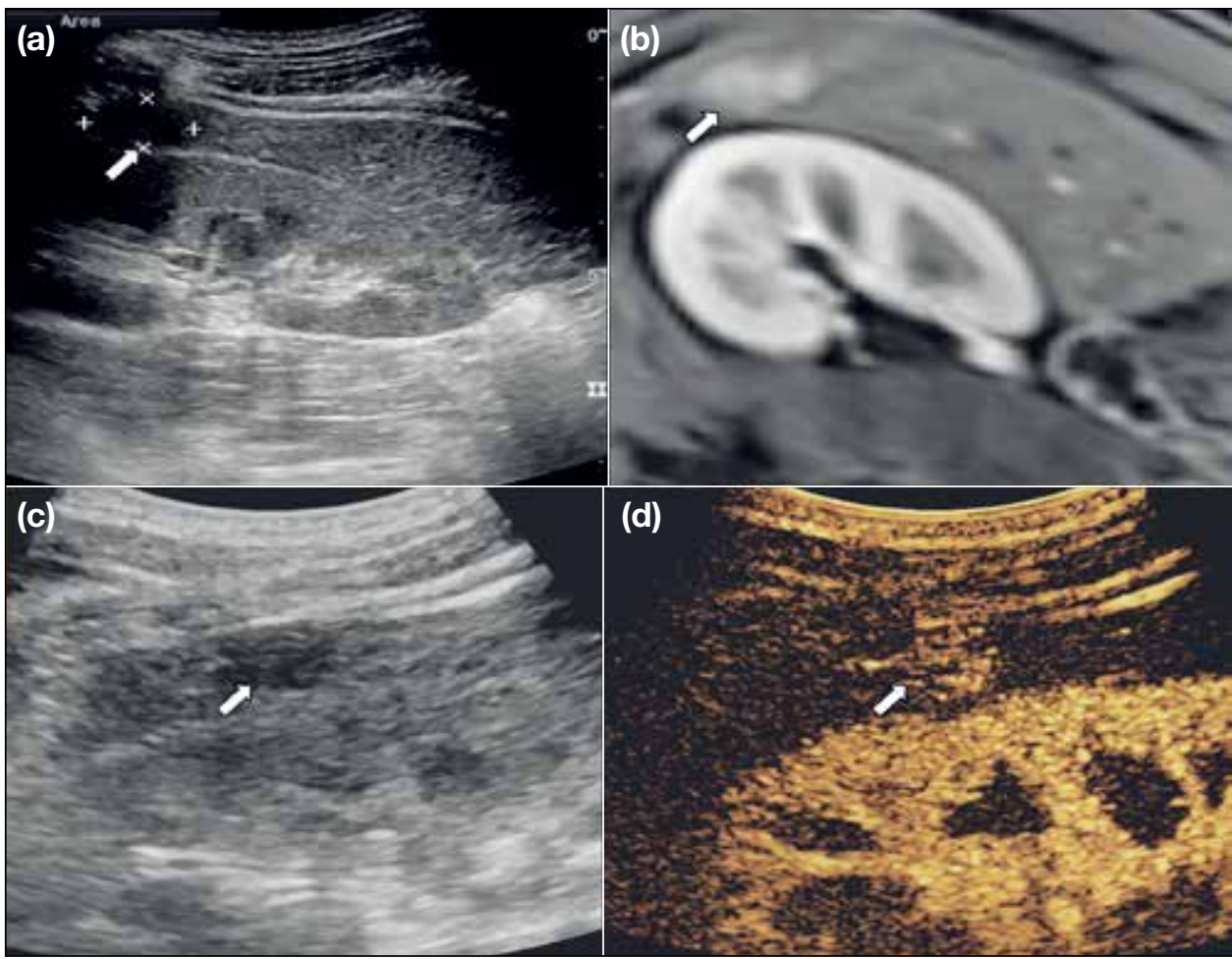

Figure 8. Contrast-enhanced ultrasound-magnetic resonance fusion images of a patient who underwent radiofrequency ablation of a $1.8-\mathrm{cm}$ segment 6 hepatocellular carcinoma (arrows). Coregistered (a) ultrasound and (b) portovenous phase magnetic resonance images of segment 6 hepatocellular carcinoma. The lesion was posteriorly located and inconspicuous on greyscale ultrasound. Compared with (c) greyscale ultrasound, the lesion was more conspicuous with early enhancement after (d) contrast-enhanced ultrasound. 


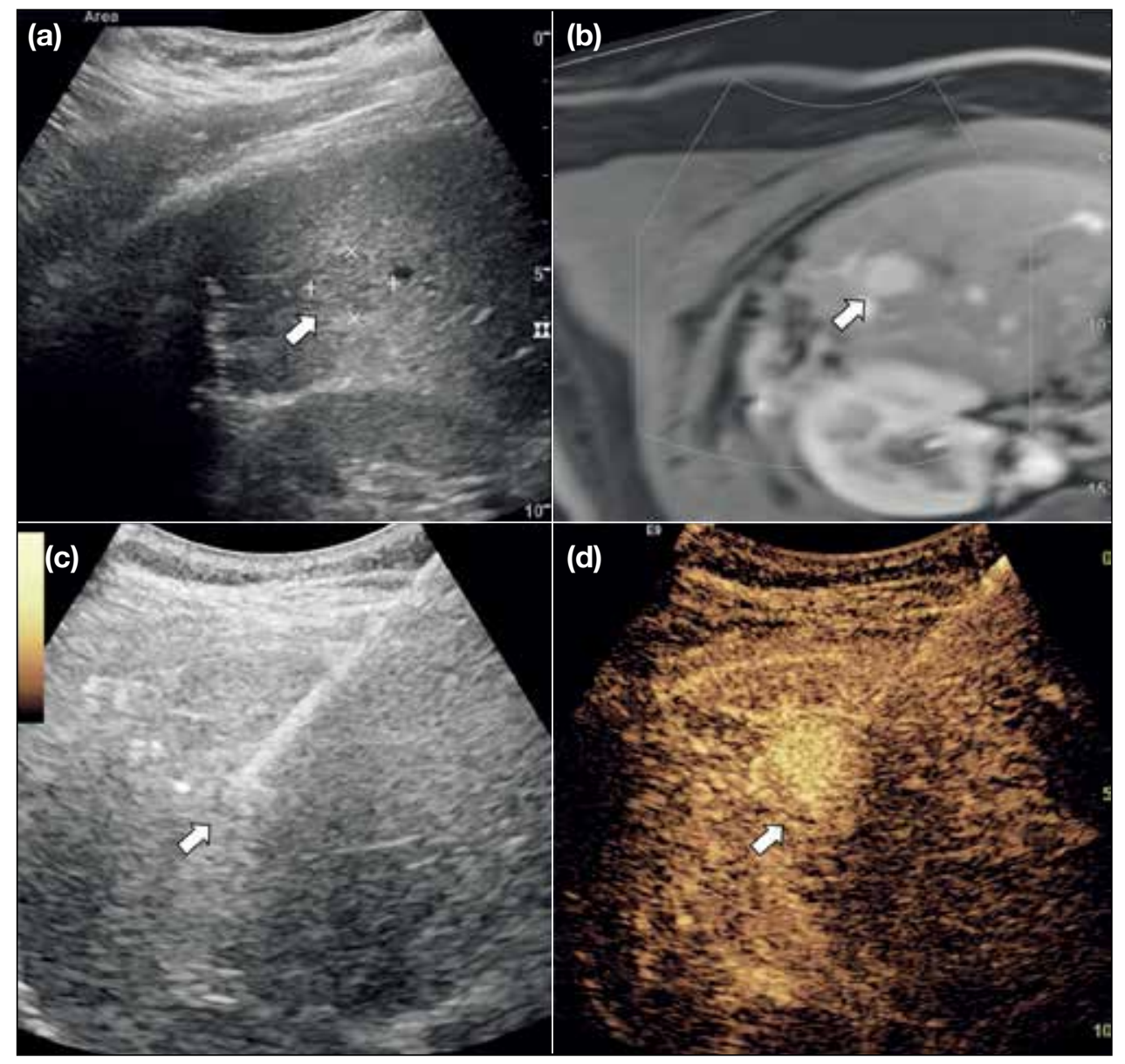

Figure 9. Contrast-enhanced ultrasound-magnetic resonance fusion images of a patient who underwent microwave ablation of segment 6 hepatocellular carcinoma (arrows). Coregistered (a) ultrasound and (b) portovenous phase magnetic resonance images of segment 6 hepatocellular carcinoma. The lesion was only slightly hypoechoic and inconspicuous on greyscale ultrasound. During microwave ablation of the target lesion, when compared with (c) greyscale ultrasound, the lesion was more conspicuous after contrast injection on (d) contrast-enhanced ultrasound.
It provides improved visualisation of the anatomy and the target lesions by exploiting the strength of contrast resolution of $\mathrm{CT}$ or MR and combining this with realtime ultrasound imaging. This allows more precise planning of needle paths, increases safety, and decreases radiation exposure. In muscle biopsy, ultrasound fusion imaging can be used to target the most inflamed tissue, potentially increasing diagnostic yield and replacing invasive surgical open biopsy. Future studies would be helpful to explore new clinical applications of ultrasound fusion imaging, such as its utility in diagnostic imaging of other organs or during surgery.

\section{REFERENCES}

1. Baad M, Lu ZF, Reiser I, Paushter D. Clinical significance of US artifacts. Radiographics. 2017;37:1408-23.

2. Lee MW.Fusion imaging of real-time ultrasonography with CT or MRI for hepatic intervention. Ultrasonography. 2014;33:227-39.

3. Ewertsen C, Săftoiu A, Gruionu LG, Karstrup S, Nielsen MB. Real time image fusion involving diagnostic ultrasound. AJR Am J Roentgenol. 2013;200:W249-55.

4. Sumi H, Itoh A, Kawashima H, Ohno E, Itoh Y, Nakamura Y, et al.
Preliminary study on evaluation of the pancreatic tail observable limit of transabdominal ultrasonography using a position sensor and CT fusion image. Eur J Radiol. 2014;83:1324-31.

5. Lee KH, Lau V, Gao Y, Li YL, Fang BX, Lee R, et al. UltrasoundMRI fusion for targeted biopsy of myopathies. AJR Am J Roentgenol. 2019 Feb 26. Epub ahead of print.

6. Burke CJ, Bencardino J, Adler R. The potential use of ultrasoundmagnetic resonance imaging fusion applications in musculoskeletal intervention. J Ultrasound Med. 2017;36:217-24.

7. Ukimura O, Mitterberger M, Okihara K, Miki T, Pinggera GM, Neuruer R, et al. Real-time virtual ultrasonographic radiofrequency ablation of renal cell carcinoma. BJU Int. 2008;101:707-11 .

8. Klauser AS, De Zordo T, Feuchtner GM, Djedovic G, Weiler RB, Faschingbauer R, et al. Fusion of real time US with CT images to guide sacroiliac joint injection in vitro and in vivo. Radiology. 2010;256:547-53.

9. Chung YE, Kim KW. Contrast-enhanced ultrasonography: advance and current status in abdominal imaging. Ultrasonography. 2015;34:3-18.

10. Park HJ, Lee MW, Lee MH, Hwang J, Kang TW, Lim S, et al. Fusion imaging-guided percutaneous biopsy of focal hepatic lesions with poor conspicuity on conventional sonography. J Ultrasound Med. 2013;32:1557-64.

11. Sartori S, Tombesi P, Macario F, Nielsen I, Tassinari D, Catellani M, et al. Subcapsular liver tumors treated with 
percutaneous radiofrequency ablation: a prospective comparison with nonsubcapsular liver tumors for safety and effectiveness. Radiology. 2008;248:670-9.

12. Patidar Y, Singhal P, Gupta S, Mukund A, Sarin SK. Radiofrequency ablation of surface $\mathrm{v} / \mathrm{s}$ intraparenchymal hepatocellular carcinoma in cirrhotic patients. Indian J Radiol Imaging. 2017;27:496-502.

13. Lee MW, Rhim H, Cha DI, Kim YJ, Lim HK. Planning US for percutaneous radiofrequency ablation of small hepatocellular carcinomas (1-3 cm): value of fusion imaging with conventional US and CT/MR images. J Vasc Interv Radiol. 2013;24:958-65.

14. Song KD, Lee MW, Rhim H, Cha DI, Chong Y, Lim HK. Fusion imaging-guided radiofrequency ablation for hepatocellular carcinomas not visible on conventional ultrasound. AJR Am J Roentgenol. 2013;201:1141-7.

15. Zhang H, Chen GY, Xiao L, Ma X, Shi L, Wang T, et al.Ultrasonic/ CT image fusion guidance facilitating percutaneous catheter drainage in treatment of acute pancreatitis complicated with infected walled-off necrosis. Pancreatology. 2018;18:635-41.

16. European Society of Radiology (ESR). Abdominal applications of ultrasound fusion imaging technique: liver, kidney, and pancreas. Insights Imaging. 2019;10:6.

17. Sidhar K, McGahan JP, Early HM, Corwin M, Fananapazir G, Gerscovich EO. Renal cell carcinomas: sonographic appearance depending on size and histologic type. J Ultrasound Med. 2016;35:311-20.

18. Helck A, D'Anastasi M, Notohamiprodjo M, Thieme S, Sommer W,
Reiser M, et al. Multimodality imaging using ultrasound image fusion in renal lesions. Clin Hemorheol Microcirc. 2012;50:7989.

19. Breen DJ, Railton NJ. Minimally invasive treatment of small renal tumors: trends in renal cancer diagnosis and management. Cardiovasc Intervent Radiol. 2010;33:896-908.

20. Andersson M, Hashimi F, Lyrdal D, Lundstam S, Hellström M. Improved outcome with combined US/CT guidance as compared to US guidance in percutaneous radiofrequency ablation of small renal masses. Acta Radiol. 2015;56:1519-26.

21. Van De Vlekkert J, Maas M, Hoogendijk JE, De Visser M, Van Schnik IN. Combining MRI and muscle biopsy improves diagnostic accuracy in subacute- onset idiopathic inflammatory myopathy. Muscle Nerve. 2015;51:253-8.

22. Rübenthaler J, Paprottka KJ, Marcon J, Reiser M, Clevert DA. MRI and contrast enhanced ultrasound (CEUS) image fusion of renal lesions. Clin Hemorheol Microcirc. 2016;64:457-66.

23. Meloni MF, Smolock A, Cantisani V, Bezzi M, D’Ambrosio F, Proiti M, et al. Contrast enhanced ultrasound in the evaluation and percutaneous treatment of hepatic and renal tumors. Eur J Radiol. 2015;84:1666-74.

24. Hakime A, Deschamps F, De Carvalho EG, Teriitehau C, Auperin A, De Baere T. Clinical evaluation of spatial accuracy of a fusion imaging technique combining previously acquired computed tomography and real-time ultrasound for imaging of liver metastases. Cardiovasc Intervent Radiol. 2011;34:338-44. 